\title{
PSEUDOTUMOR ENDOBRONQUIAL ASOCIADO A ASPERGILOSIS. A PROPÓSITO DE UN CASO. HOSPITAL TEODORO MALDONADO CARBO
} ENDOBRONCHIAL PSEUDOTUMOR ASSOCIATED WITH ASPERGILLOSIS. ABOUT A CASE. HOSPITAL TEODORO MALDONADO CARBO

PSEUDOTUMOR ENDOBRONQUIALASSOCIADO COM ASPERGILOSE. UM OBJETIVO DE UM CASO. HOSPITAL TEODORO MALDONADO CARBO

\section{ZOILA TERÁN JIMÉNEZ1', MARÍA JOSÉ ULLOA SILVA', FLAVIO ESCOBAR SÁNCHEZ1', SANDRA LORENA RODRÍGUEZ ZAMBRANO'}

\author{
${ }^{1}$ Hospital Teodoro Maldonado Carbo, Guayaquil, Ecuador
}

\begin{abstract}
Resumen varios estudios, un cuerpo extraño que había pasado inadvertido para el paciente durante 3 años. La presencia de un cuerpo extraño endobronquial en adultos puede ser de difícil diagnóstico, cuando no es referido el antecedente de aspiración y a encontrarse asociado a otras patologías. Se expone un caso clínico de difícil diagnóstico y alta complejidad ante la presencia de tumoración endobronquial que inicialmente por broncoscopía fue definida como aspergilosis endobronquial; presentando posteriormente neumonía severa y fue en un segundo intento de biopsia para evitar cirugía, que se logra remover la lesión que resultó ser una semilla de tamarindo que el paciente la había aspirado años atrás; con la posterior complicación de sepsis e insuficiencia respiratoria que requirió asistencia ventilatoria mecánica y antibióticos de amplio espectro; cursando posteriormente con recuperación total. En conclusión ante la presencia de una imagen endobronquial se debe tener en cuenta la posibilidad de cuerpo extraño dentro de los diagnósticos diferenciales; siendo la broncoscopía el método ideal para su diagnóstico definitivo y evitando así realizar intervenciones quirúrgicas.
\end{abstract}

PALABRAS CLAVE: broncoscopía, cuerpos extraños, aspergilosis.

Abstract

The term of endobronchial pseudotumor is mentioned in the presence of an image that occupied the lumen of a bronchus that turned out to be, after several studies, a foreign body that had gone unnoticed by the patient for 3 years. The presence of an endobronchial foreign body in adults can be difficult to diagnose, when the antecedent of aspiration is not mentioned it can be associated with other diseases. We present a case of difficult diagnosis and high complexity in the presence of an endobronchial tumor that was initially defined as endobronchial aspergillosis by bronchoscopy; presenting later severe pneumonia and it was in a second attempt of getting a biopsy to avoid surgery, that it is possible to remove the lesion that turned out to be a tamarind seed that the patient had aspired years ago; with the subsequent complication of sepsis and respiratory failure the patient required mechanical ventilation and broad-spectrum antibiotics; later evolving to full recovery. In conclusion, in the presence of an endobronchial image, the possibility of a foreign body should be considered in the differential diagnoses; Bronchoscopy being the ideal method for its definitive diagnosis and thus avoiding surgical interventions. KEYWORDS: bronchoscopy, foreign bodies, aspergillosis.

Resumo

O termo de pseudotumor endobrônquica é mencionado na presença de uma imagem ocupada pela luz de um brônquio acabou por ser, após vários estudos, um corpo estranho que tinha passado despercebido pelo paciente por 3 anos. A presença de corpo estranho endobrônquico em adultos pode ser difícil de diagnosticar, quando 0 antecedente da aspiração não é mencionado e está associado a outras patologias. um caso de difícil diagnóstico e complexidade elevada na presença de tumor endobrônquico inicialmente broncoscopia foi definida como aspergilose endobrônquica é exposto; posteriormente mostrando pneumonia grave e estava em uma segunda tentativa de evitar a biópsia cirurgia, conseguido remover a lesão provou ser uma semente de tamarindo 0 paciente tinha desenhado anos atrás; com a complicação subsequente de sepse e insuficiência respiratória que exigia ventilação mecânica e antibióticos de amplo espectro; mais tarde estudando com recuperação total. Em conclusão, na presença de uma imagem endobrônquica, a possibilidade de um corpo estranho deve ser levada em consideração nos diagnósticos diferenciais; A broncoscopia é o método ideal para o diagnóstico definitivo, evitando intervenções cirúrgicas.

PALAVRAS-CHAVE: broncoscopia, corpos estranhos, aspergilose. 
INTRODUCCIÓN

La presencia de un cuerpo extraño endobronquial es, una entidad poco frecuente en adultos, a diferencia de la edad pediátrica;1,2 resulta difícil diagnosticarlo tempranamente por hallazgos clínicos cuando la aspiración ha pasado inadvertida para el paciente y más aún, cuando la lesión está en asociación a aspergilosis, pudiendo ser confuso para establecer un diagnóstico definitivo por imagenología. ${ }^{3,4}$

La broncoscopía es el procedimiento estándar para el diagnóstico y extracción de un cuerpo extraño sin necesidad de recurrir a intervenciones quirúrgicas. El procedimiento es generalmente seguro y bien tolerado, la extracción puede realizarse con broncoscopía flexible o rígida siendo imperativo que el broncoscopista esté familiarizado con las dos técnicas y con el equipo adecuado. $^{5-7}$

Se realiza revisión de la literatura médica y pese a que existen casos publicados en la literatura mundial, en Ecuador los casos reportados son muy escasos pudiendo ser en ocasiones subdiagnosticados, retrasando el diagnóstico definitivo e influyendo en el pronóstico del paciente. Se expone el caso de un hombre de 66 años quien presentó una lesión pseudotumoral en bronquio principal izquierdo que por broncoscopía inicialmente, fue definida como aspergilosis endobronquial, presentando posteriormente neumonía severa. En un segundo estudio broncoscópico se logró remover la lesión que resultó ser semilla de tamarindo que el paciente la había aspirado 3 años atrás; complicándose con insuficiencia respiratoria y sepsis mejorando con esquema amplio de antibióticos y apoyo ventilatorio mecánico.

CASO CLÍNICO

Paciente masculino de 66 años de edad; ingresa al hospital Teodoro Maldonado Carbo el 24 de agosto del 2015, refiriendo ser diabético, hipertenso y tres meses antes haber sido diagnosticado de aspergilosis endobronquial tras habérsele realizado biopsia por broncoscopía que mostraba una lesión tumoral endobronquial, en bronquio principal izquierdo que ocupaba el $90 \%$ de la luz y por lo que recibía tratamiento con itraconazol $400 \mathrm{mg}$ diarios.

Paciente ingresa en mal estado general con fiebre de $40^{\circ} \mathrm{C}$, una semana de evolución, tos con expectoración verdosa, con regular mecánica respiratoria, presentando importante pérdida de panículo adiposo, (3Kg en 3 meses). A la auscultación: roncus y estertores crepitantes en hemitórax izquierdo. Resto del examen físico normal.Se inicia seguimiento del paciente, obteniendo exámenes de laboratorio: de hemoglobina en 10.1 (14,00-18,00 g/dl), hematocrito en $34(42,00-52,00 \%)$, leucocitos de 27.600 (4,50-10,00

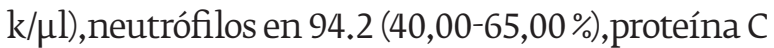
reactiva en $22,4(<1,00-3,00)$, procalcitonina de 3 (0.00-0.500), tiempo de protrombina en 16,6 (10.814,5 SEG.) y tiempo parcial de tromboplastina de 40,3 (20,00-33,3 SEG.)

En la tomografía axial computarizada (TAC) de tórax se observa tumoración endobronquial a la entrada de bronquio fuente izquierdo, con densidad de tejido blando, (Figura 1) y lesión condensativa y atelectasia en lóbulo inferior izquierdo, (Figura 2).

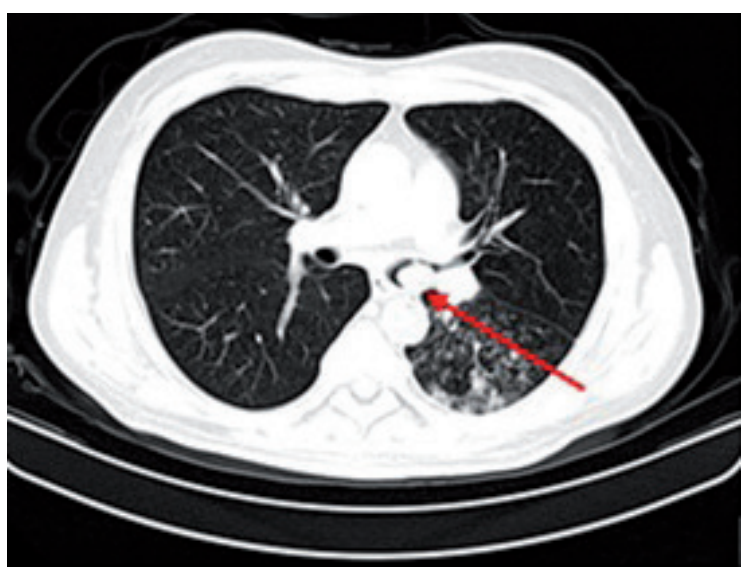

Figura 1. Imagen endobronquial, en bronquio principal izquierdo.

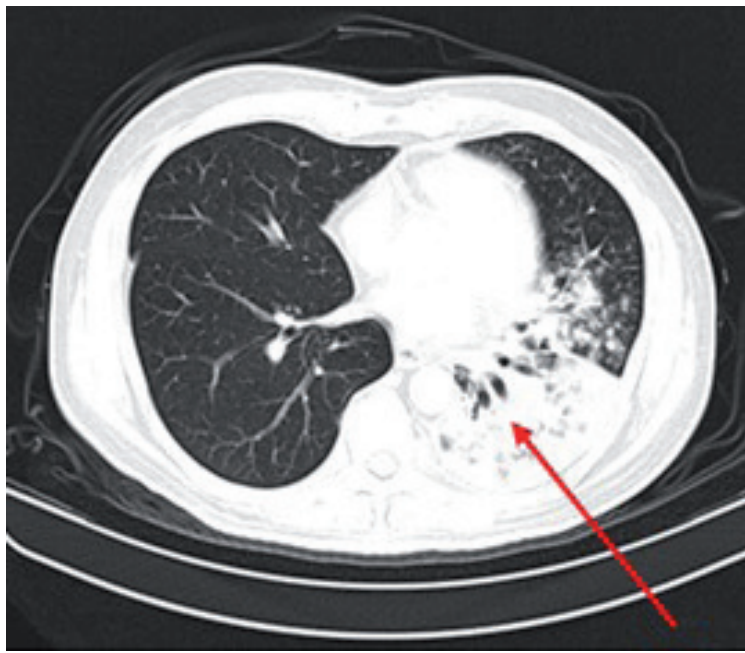

Figura 2. Lesión condensativa en lóbulo inferior izquierdo.

En el examen de esputo se encuentra citología negativa, baciloscopias negativas; cultivo de hongos negativos y cultivo de esputo para bacterias (enterobactercloacae) negativos. 
Catalogado como proceso infeccioso con neumonitis postobstructiva severa, se inicia esquema con antibióticos y antimicóticos consiguiendo luego de 13 días de internación, una buena respuesta clínica al tratamiento instaurado, por lo que fue dado de alta con itraconazol $400 \mathrm{mg}$ diarios.

En un nuevo control tomográfico, se observa persistencia de tumor endobronquial, por lo que se programa un nuevo estudio endoscópico con biopsia el 23 de noviembre del 2015, en el cual se encontró lesión tumoral café-nacarada que ocupaba el $90 \%$ de la entrada del bronquio principal izquierdo, (Figura 3). Por sus características fue difícil su abordaje para biopsiarla; notándose material necrótico y purulento que provenía del lóbulo inferior, desprendiéndose de su pedículo y alojándose en el bronquio lobar inferior; decidiéndose extraerlo con broncoscopio rígido(Figura 4).

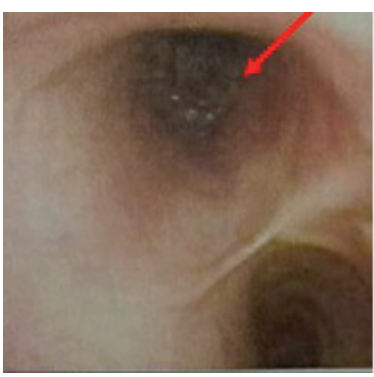

Figura 3. Obstrucción de la luz del bronquio izquierdo con material necrótico.

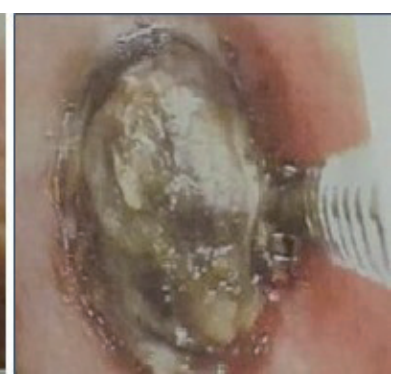

Figura 4. Extracción de lesión tumoral café-nacarada con broncoscopio rígido.

Durante la broncoscopía rígida, se extrae un tumor de 1,6 cm, color café de característica pétrea sin vasculatura; al manipularlo impresiona como cuerpo extraño, (Figura 5); enviado el material (cuerpo extraño) a patología y laboratorio de microbiología para su procesamiento, cuyos resultados fueron: tejido vegetal (cuerpo extraño), (Figura 6) y cultivos negativos.

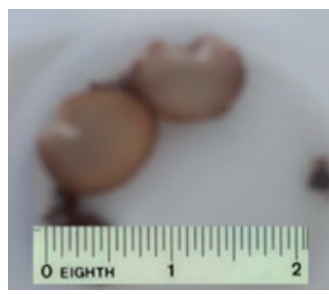

Figura 5. Cuerpo extraño, semilla de tamarindo.

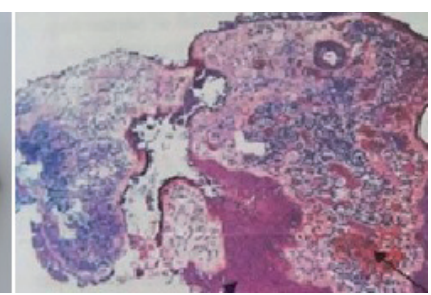

Figura 6. Bronquitis aguda y crónica con erosión y metaplasia escamosa, tejido vegetal (cuerpo extraño).
Por lo difícil del procedimiento y cuadro clínico muy comprometido, hemodinámicamente paciente requirió manejo en unidad de cuidados intensivos con apoyo de ventilación mecánica, presentando neumonía severa y sepsis; permaneciendo 9 días en esta unidad, recibiendo un esquema amplio de antibióticos y antimicóticos, completando su tratamiento en el área de neumología con un tiempo de hospitalización de 7 días; luego es dado de alta al encontrarse asintomático y con buena mecánica respiratoria. El laboratorio demostraba ausencia de leucocitosis; la tomografía, aún presentaba lesiones en hemitorax izquierdo, lóbulo inferior.

Al confirmarse la presencia de un cuerpo extraño en la vía aérea, el paciente recordó haber tenido hace 3 años un episodio de asfixia con una semilla de tamarindo. Desde el inicio de los síntomas hasta la detección del cuerpo extraño, transcurrieron 8 meses. En la actualidad el paciente continúa en evaluación periódica y hasta la última visita se encontraba asintomático.

\section{DISCUSIÓN}

Se menciona el término de pseudotumor endobronquial ante la presencia de una imagen que ocupaba el bronquio fuente izquierdo que posteriormente resultó ser un cuerpo extraño. El desconocimiento del antecedente de aspiración y la manifestación clínica inespecífica hacen de esta entidad una patología insospechada. En el presente reporte de caso, el paciente permaneció asintomático por un largo tiempo hasta que el cuerpo extraño produjo cambios debido a la obstrucción e inflamación del orificio bronquial, así como lo menciona Qureshi. ${ }^{1}$

En un estudio realizado en pacientes geriátricos, los síntomas más comunes detectados fueron tos, expectoración, disnea y hemoptisis, lo cual se puede observar en muchas patologías respiratorias. En consecuencia, entre el 80 al $90 \%$ de los pacientes fueron mal diagnosticados con neumonía o sospecha de cáncer de pulmón durante meses o años, hasta que la broncoscopía flexible confirmó correctamente la presencia de un cuerpo extraño. ${ }^{1,2,8}$

La tomografía computarizada no permite reconocer con exactitud la naturaleza de la lesión y de acuerdo a las características del cuerpo extraño, la orientación diagnóstica varía. Los cuerpos metálicos por su excelente contraste a los rayos 
$\mathrm{X}$, son fácilmente diagnosticados y localizados, y su sospecha es de inmediato; ${ }^{9}$ por el contrario los cuerpos extraños vegetales no son visibles a los rayos $\mathrm{X}$ y en contacto con las secreciones bronquiales, pueden aumentar su tamaño, ocasionando una mayor obstrucción, haciendo difícil su extracción. ${ }^{10}$

En este reporte de caso, el diagnóstico inicial por biopsia de aspergilosis endobronquial fue impreciso para establecer un diagnóstico definitivo por lo que después de mejorar el cuadro infeccioso se programó un segundo estudio broncoscópico.La aspergilosis endobronquial ha sido asociada a cuerpo extraño y descrita por la literatura médica como una patología poco común de aspergilosis pulmonar. Ma JE y colaboradores estudiaron 10 casos de aspergilosis endobronquial asociada a neoplasia, tuberculosis, resección pulmonar, siendo un solo caso por cuerpo extraño. ${ }^{11-13}$

La broncoscopía es considerada el método esencial para el diagnóstico y manejo de las lesiones endobronquiales. ${ }^{5-7}$ La localización más frecuente de un cuerpo extraño por orden decreciente de frecuencia son las siguientes: Bronquio principal derecho (60-65\%), bronquio principal izquierdo (25-30\%) y subglotis-tráquea $(9-12 \%) .{ }^{3}$ En el paciente fue en el bronquio fuente izquierdo.

En la actualidad existen estudios que debaten el uso de la broncoscopía flexible vs el broncoscopio rígido. Fang YF y colaboradores,indican en su estudio que la broncoscopía flexible es eficaz para el diagnóstico y la eliminación de cuerpos extraños en la vía aérea inferior en adultos, teniendo un éxito en 85 pacientes (90\%) y sin diferencia entre aspiraciones agudas y crónicas. ${ }^{5,7}$ Del mismo modo la literatura sugiere a la broncoscopía flexible como el estudio de primera línea para pacientes geriátricos, especialmente aquellos con historial de aspiración y neumonía de origen desconocido. ${ }^{8}$ En el presente reporte de caso, en el segundo estudio broncoscópico hubo complejidad al intentar biopsiar la lesión, que resultó ser un cuerpo extraño por lo que fue necesario y urgente extraerla por broncoscopía rígida.

En una serie de casos, Rodríguez $\mathrm{H}$ y colaboradores exponen que cuanto más tiempo permanece el cuerpo extraño en el árbol bronquial mayor es la posibilidad de complicaciones. ${ }^{14-17}$
CONCLUSIÓN

El hallazgo de un cuerpo extraño endobronquial en adultos representa una entidad inusual; mas aún si se encuentra asociado a un cuadro de aspergilosis. Los autores destacan la importancia de la broncoscopía en el diagnóstico y manejo de esta patología. Siendo imperativo que el broncoscopista esté familiarizado con las dos técnicas broncoscópicas (flexible y rígida) pudiendo así evitar procedimientos quirúrgicos, quedando reservado éste como último recurso.

\section{REFERENCIAS BIBLIOGRÁFICAS}

1. Qureshi RA, Soorae AS. Foreign body in tracheal bronchus simulating bronchogenic cancer. Eur J CardiothoracSurg. 2001 Sep;20 (3):639-41.

2. Sánchez-Font A, Vollmerb I, Gracia M. P, Gayete A y Curull V. Falsas imágenes de tumoración endobronquial por tomografía computarizada. Rev Clin Esp. 2008; 208(9):444-6

3. Calvo-Âlvarez Y, Hernández-Vila Y, Fernández García S, Sarduy Paneque M, Córdova Rozas E. Cuerpo extraño endobronquial. Presentación de un caso. Cuerpo Extraño Endobronquial. Presentación de un Caso. Revista Habanera de Ciencias Médicas 2013; 12(4):542-548

4. Domingo Morera JA, Torres Nuez J, Sánchez Santos P, Mateo Lázaro ML, Bello Dronda S, Martínez Sanz G. Intrabronchialforeignbody in adults: usefulnessofcomputerizedtomography. Arch Bronconeumol. 1997 Apr;33(4):201-3. PubMed; PMID9280565

5. Fang YF, Hsieh MH, Chung FT, Huang YK, Chen GY, Lin SM, et al. Flexible Bronchoscopy with Multiple Modalities for Foreign Body Removal in Adults. Biomed Res Int. 2015 Mar 13;10(3):e0118993. doi: 10.1371/journal.pone.0118993. PubMed; PMID 26609535; PubMed Central PMCID: PMC4358882.

6. Ganie FA, Wani ML, Ahangar AG, Lone GN, Singh S, Lone H, Wani SN. The Efficacy of Rigid Bronchoscopy for Foreign Body Aspiration. Bull Emerg Trauma. 2014 Jan;2(1):52-4.PubMed; PMID: 27162864 5, PubMed Central PMCID: PMC4771260.

7. Gupta S, Bhalotra B, Jain N.. Spectrum of Intrabronchial Mass Lesions and Role of Flexible Bronchoscopy in Their Diagnosis: A Series of 74 Cases. Indian J Chest Dis Allied Sci. 2010 Apr-Jun;52(2):79-82. PMID: 20578399

8. Lin L, Lv L, Wang Y, Zha X, Tang F, Liu X. The clinical features of foreign body aspiration into the lower airway in geriatric patients.Clin IntervAging. 2014 Sep 24;9:1613-8. doi: 10.2147/CIA.S70924. PubMed; PMID: 25284994

9. Carranza M, Callejas MA, Freixinet JL, Gimferrer JM, Sánchez-Lloret J, Xaubet A. Pseudotumorendobronquial como manifestación de la aspiración de 
un cuerpo extraño. Archivos de Bronconeumología.1986; 22(5): 243-244.

10. Holinger PH, Holinger LD. Use of the open tube bronchoscope in the extraction of foreign bodies. Chest 1978 Mayo; 73 (5):721-724.

11. Jung SW, Kim MW, Cho SK, et al. A Case of Endobronchial Aspergilloma Associated with Foreign Body in Immunocompetent Patient without Underlying Lung Disease. TubercRespir Dis. 2013 May;74(5):2314. doi: 10.4046/trd.2013.74.5.231. PubMed; PMID: 23750172

12. Ma JE, Yun EY, Kim YE et al. Endobronchial aspergilloma: report of 10 cases and literature review. Yonsei Med J. 2011 Sep;52(5):787-92. doi: 10.3349/ ymj.2011.52.5.787. PubMed; PMID: 21786444

13. Fernández González S, Suarez PereiroM.J, Bollo de MiguelE, Alonso-Orcajo N. Aspergilomaendobronquial. Rev ClinEsp 2013;213(7):71-2. doi: 10.1016/j. rce.2013.02.009
14. Chen CH, Lai CL, Tsai TT, Lee YC, Perng RP. Foreign body aspiration into the lower airway in Chinese adults. Chest. 1997 Jul;112(1):129-33. PubMed; PMID: 9228368

15. Zissin R, Shapiro-Feinberg M, Rozenman J, Apter $S$, Smorjik J, Hertz M. CT findings of the chest in adults with aspirated foreign bodies. EurRadiol. 2001;11(4):606-11. PubMed; PMID: 11354755.

16. Rodríguez H, Cuestas G; Botto H, et al. Demora en el diagnóstico de un cuerpo extraño en la vía aérea en los niños: Serie de casos. Arch. argent. Pediatr.2013jun;111(3):69-73.

17. Yanowsky Reyes G, Aguirre Jáuregui O, Rodríguez Franco E, et al. Cuerposextrañosenvíasaéreas. Archivos De Medicina. 2013, Apr, 9(2): 1-7. doi: 10.3823/096. 